Running head: CATEGORY FLUENCY IN SCHIZOTYPY

\title{
Category fluency in psychometric schizotypy: How altering emotional valence and cognitive load affects performance
}

\footnotetext{
${ }^{1}$ Department of Psychology, Indiana University- Purdue University Indianapolis, Indianapolis, IN, United States

${ }^{2}$ Department of Psychology, Louisiana State University, Baton Rouge, LA, United States
}

\author{
${ }^{*}$ Corresponding Author: Kyle S. Minor; IUPUI School of Science, Department of Psychology, \\ LD 124, 402 N. Blackford St., Indianapolis, IN, 46202; Phone: (317) 274 -2933; Fax: (317) 274- \\ 6756; email: ksminor@iupui.edu
}

This is the author's manuscript of the article published in final edited form as:

Minor, K. S., Luther, L., Auster, T. L., Marggraf, M. P., \& Cohen, A. S. (2015). Category fluency in psychometric schizotypy: how altering emotional valence and cognitive load affects performance. Cognitive Neuropsychiatry, 20(6), 542-550. http://doi.org/10.1080/13546805.2015.1116441 


\begin{abstract}
Introduction. In clinical high-risk populations, category fluency deficits are associated with conversion to psychosis. However, their utility as clinical risk markers is unclear in psychometric schizotypy, a group experiencing schizophrenia-like traits that is at putative high risk for psychosis.

Methods. We examined whether introducing affective or cognitive load, two important stress vulnerability markers, altered category fluency performance in schizotypy $(n=42)$ and nonschizotypy $(n=38)$ groups. To investigate this question, we developed an experimental paradigm where all participants were administered category fluency tests across baseline, pleasant valence, unpleasant valence, and cognitive load conditions.

Results. Compared to the non-schizotypy group, those with schizotypy performed significantly worse in pleasant and unpleasant valence conditions, but not cognitive load or baseline fluency tests.

Conclusions. This study demonstrated the role of affect - but not cognitive load — on category fluency in psychometric schizotypy, as group differences only emerged once affective load was introduced. One explanation for this finding is that semantic memory may be unimpaired under normal conditions in psychometric schizotypy, but may be compromised once affective load is presented. Future studies should examine whether fluency deficits - particularly when affect is induced - predict future conversion to psychosis in psychometric schizotypy cohorts.
\end{abstract}

Keywords: emotion; affect; cognition; category fluency; schizotypy 
In clinical high-risk populations, category fluency deficits - among the largest and most stable neurocognitive impairments in schizophrenia (Bokat \& Goldberg, 2004; Szoke et al., 2008) — have shown promise as a clinical risk marker for psychosis (Becker et al., 2010; Eastvold, Heaton, \& Cadenhead, 2007; Hawkins et al., 2004). The evidence is less clear, however, for using category fluency deficits as a clinical risk marker in psychometric schizotypy — a group at putative high risk for psychotic and other psychiatric disorders (Lenzenweger, 2006; Meehl, 1990; see Chun, Minor, \& Cohen, 2013). One strategy for uncovering category fluency deficits in psychometric schizotypy is to administer tests with experimental manipulations; ideally, manipulations would be designed by modifying stimuli to highlight other potential risk markers. Two proposed pathways that may increase risk for conversion to psychosis include: 1) an affective pathway, associated with higher levels of positive-like traits and;2) a cognitive pathway, associated with greater negative-like traits (Myin-Germeys, Krabbendam, Jolles, Delespaul, \& van Os, 2002; Morrens et al., 2007; see Myin-Germeys \& van Os, 2007). Thus, designing a category fluency paradigm that alters affective and cognitive stimuli holds the possibility of elucidating which people with psychometric schizotypy may be at the highest risk for later conversion.

For affective manipulations, category fluency tests designed to alter emotional valence have already been developed (Rossell, 2006). These tests require participants to name words associated with happiness (e.g., pleasant valence) and fear (e.g., unpleasant valence). In an initial study, Rossell (2006) observed that both schizophrenia and control groups scored significantly lower on emotionally-valenced category fluency tests compared to standard tests (e.g., animals, food). To our knowledge, these tests have not been utilized in a psychometric schizotypy 
population. In this study, our aim was to examine whether the schizotypy group demonstrated a sharper decline in fluency from baseline to affective tests than the non-schizotypy group. Unlike affective tests, category fluency tests have not been previously designed with cognitive manipulations. In this study, we created cognitive manipulations by adhering to cognitive load theory. Cognitive load theory posits that increasing load reduces one's available cognitive resources due to working memory's limited capacity-making it more difficult to adequately attend to two tasks at the same time (Sweller, 1983). Although the cognitive load effect diminishes performance in healthy adults (Ruthruff, Pashler, \& Klaassen, 2001; van Merrienboer \& Sweller, 2010), it appears to be more pronounced in schizophrenia-spectrum populations: Schizophrenia and schizotypal personality disorder cohorts have demonstrated increased difficulty, compared to controls, switching between tasks (Nuechterlein, Pashler, \& Subotnik, 2006) and performing a dual digit span-visual perception test (Harvey, Reichenberg, Romero, Granholm, \& Siever, 2006). In this study, we compared category fluency performance in psychometric schizotypy and non-schizotypy groups across baseline and cognitive conditions.

In the current study, we had two primary aims. First, category fluency performance in schizotypy and non-schizotypy groups was examined across baseline, pleasant valence, unpleasant valence, and cognitive load conditions. We expected all participants would perform significantly worse on affective and cognitive fluency tests compared to baseline tests, but that performance in the schizotypy group would decline more sharply. We also hypothesized that the schizotypy group would: a) perform significantly worse overall on category fluency tests and b) exhibit significantly worse combined performance (i.e., fluency plus accuracy on the cognitive task) in the cognitive load condition. Second, we expected that, within the schizotypy group, positive and disorganized traits would be associated with worse fluency in pleasant and 
unpleasant valence conditions, whereas negative traits would be linked to worse performance in baseline and cognitive load conditions.

\title{
Method \\ Participants
}

Participants were recruited from a university in the Southeastern United States via email to complete a schizotypy questionnaire in exchange for course credit and entry into a gift card drawing $(n=1,296)$. Schizotypy $(z$-score $>1.65$ above mean on positive, negative, or disorganized subscales; $n=126)$ and non-schizotypy groups ( $z$-score $<$ mean on each subscale; $n$ $=380$ ) were determined from questionnaire responses using gender and ethnicity derived means. Those meeting criteria were invited to the laboratory for further testing. Additional inclusion criteria were: 1$)$ age 18-30 ( $n$ excluded $=1)$ and 2$)$ complete data for all category fluency tests $(n$ excluded $=5$ ). The final sample consisted of 42 schizotypy and 38 non-schizotypy subjects (see Table 1 for demographic comparisons). All study procedures were approved by an internal institutional review board.

\section{[INSERT TABLE ONE HERE]}

\author{
Measures \\ Schizotypal Personality Questionnaire-Brief Revised (SPQ-BR) \\ The SPQ-BR (Cohen, Matthews, Najolia, \& Brown, 2010) consists of 34 Likert-scale items (1 = \\ Strongly Disagree, 5 = Strongly Agree) and was used to determine group status. The SPQ-BR \\ was developed from the Schizotypy Personality Questionnaire (SPQ; Raine, 1991), has a similar \\ three factor structure (positive, negative, disorganized), and has demonstrated strong
}


psychometric properties (see Cohen et al., 2010). In previous studies, individuals with elevations on psychometric questionnaires - like the SPQ-BR — have exhibited increased risk for psychotic and other psychiatric disorders (Cohen \& Najolia, 2011; Gooding, Tallent, \& Matts, 2005; Kwapil, Miller, Zinser, Chapman, \& Chapman, 1997).

\section{Category fluency tests}

Category fluency tests were administered across four conditions (baseline, pleasant valence, unpleasant valence, cognitive load). Subject responses were checked for category accuracy by a Research Assistant and the first author of this study, with the first author making final determinations for discrepancies. Only valid responses were included in fluency scores. Following Rossell's (2006) protocol for affective valence tests, participants were given one minute to name examples of words associated with happiness (pleasant valence) and fear (unpleasant valence). Examiners provided one example during administration instructions (pleasant valence: 'dancing', unpleasant valence: 'crocodile').

Baseline and cognitive load conditions consisted of fruit and vegetable category fluency tests. In the cognitive load condition, participants completed a category fluency test while simultaneously performing a 1-back task. The 1-back task consisted of 30 consecutive shapes; participants were instructed to press ' $S$ ' if the shape was the same as the previous shape or ' $L$ ' if the shape was not the same as the previous shape. Accuracy and a composite score (i.e., combining fluency and accuracy) on the 1-back task were recorded in addition to fluency. Fruit and vegetable tests were counter-balanced across baseline and cognitive load conditions. To control for differences between fruit and vegetable tests, we approximated normative data by first administering them with and without cognitive load to 61 undergraduate students - who 
completed tests for course credit. We observed that scores were approximately $25 \%$ greater on fruit (baseline: $M=13.55$ [3.05], cognitive: $M=10.03$ [3.66]) compared to vegetable tests, (baseline: $M=12.07$ [4.71], cognitive: $M=8.00$ [2.39]) in both conditions. In the current study, 1.5 and 2 points were added to fluency scores for all participants completing vegetable tests in baseline and cognitive tasks, respectively. Corrected and uncorrected mean scores (i.e., scores without points added) are listed in Table 2.

\section{Statistical Analyses}

A two-way, mixed repeated measures analysis of variance (ANOVA) was conducted to examine hypotheses, with group (schizotypy, non-schizotypy) and condition (baseline, pleasant valence, unpleasant valence, cognitive load) as independent variables and fluency scores as the dependent variable. Individual condition main effects were examined by comparing scores on experimental fluency tests to baseline fluency across the sample using dependent-samples $t$-tests. Between group differences were followed up using independent-samples $t$-tests. In the cognitive condition, accuracy on the 1-back task and a composite score (providing equal weight to fluency and accuracy) were also compared using independent-samples $t$-tests. Finally, bivariate correlations were computed within the schizotypy group to investigate if schizotypy traits were associated with fluency. Alpha levels were set at .05 for all analyses. Outliers were reduced to within 3 SDs of the mean.

\section{Results}

\section{Performance across category fluency conditions}


Means and standard deviations for all category fluency tests are listed in Table 2. The ShapiroWilk test indicated fluency data was normally distributed. Mauchly's test showed that the assumption of sphericity had been violated, $\chi^{2}(5)=0.82, p=0.008$. Thus, degrees of freedom were corrected using the Greenhouse-Geisser estimate $(\epsilon=0.88)$. The results of the repeatedmeasures ANOVA indicated a significant main effect for condition, $F(2.64,76)=28.82, p<$ 0.01. This main effect was significant when unpleasant valence, $F(1,78)=42.17, p<0.01$, and cognitive load, $F(1,78)=15.98, p<0.01$, were compared to the baseline condition. No significant effect was found when pleasant valence and baseline fluency were compared, $F$ (1, 78) $=0.42, p=0.26$.

A significant overall group X condition interaction was observed, $F(2.64,76)=2.18, p=$ 0.049. Compared to baseline, trend level group X condition interactions were observed for pleasant, $F(1,78)=2.18, p=0.07$, and unpleasant valence, $F(1,78)=1.99, p=0.08$, but not cognitive load, $F(1,78)=0.22, p=0.32$ (see Figure 1). Significant main effects were found between groups in pleasant, $t(78)=1.77, p=0.04$, and unpleasant valence conditions, $t(78)=$ $1.94, p=0.03$, with the schizotypy group exhibiting worse performance in both. No significant group differences were observed in baseline, $t(78)=0.19, p=0.43$, or cognitive load conditions, $t(78)=-0.28, p=0.39$. Accuracy, $t(78)=0.29, p=0.39$, and composite cognitive scores, $t(78)=$ $-0.09, p=0.47$, on the cognitive load test did not vary by group.

[INSERT TABLE ONE HERE]

[INSERT FIGURE ONE HERE]

Correlations between schizotypy traits and category fluency test performance 
Within the schizotypy group, there was variability for positive $(M=1.27$ [0.89], range: $-0.83-$ 3.21), negative $(M=1.28$ [1.10], range: $-1.34-3.23)$, and disorganized traits $(M=1.41[0.58]$, range: $-0.12-2.66)$. Correlations revealed a significant inverse association between positive schizotypy and fluency in the unpleasant valence condition, $r(42)=-0.34, p=0.03$. Significant relationships were not observed between positive schizotypy and pleasant valence, $r(42)=-0.23$, $p=0.14$, cognitive load, $r(42)=-0.18, p=0.27$, or baseline fluency, $r(42)=-0.27, p=0.08$.

Disorganization traits were significantly associated with baseline fluency, $r(42)=0.35, p=0.02$, and exhibited trend-level associations with unpleasant valence, $r(42)=-0.27, p=0.09$, and cognitive load fluency, $r(42)=0.30, p=0.05$, but not fluency in the pleasant valence condition, $r(42)=0.09, p=0.57$. Fluency and negative traits were not associated in baseline, $r(42)=-0.05$, $p=0.77$, pleasant valence, $r(42)=-0.17, p=0.27$, unpleasant valence, $r(42)=0.00, p=0.98$, or cognitive load conditions, $r(42)=-0.25, p=0.11$.

\section{Discussion}

The primary aim of this study was to examine how affective valence and cognitive load influenced category fluency performance in psychometric schizotypy. Compared to a nonschizotypy group, significant group differences only emerged once pleasant and unpleasant valence was introduced. Precedence for this finding has been provided by formal thought disorder studies, where significantly larger increases in disorganization have been observed in psychometric schizotypy groups from baseline to unpleasant valence conditions (Kerns \& Becker, 2008; Minor, Cohen, Weber, \& Brown 2011; but also see Minor \& Cohen, 2010). Category fluency tests tap into a range of neurocognitive areas and are commonly used to assess semantic memory (Neill, Gurvich, \& Rossell, 2014)—an area also heavily involved in formal 
thought disorder (Kerns \& Berenbaum, 2002). Thus, retrieving words from semantic memory may be unimpaired under normal circumstances in psychometric schizotypy, but may become compromised once affective load is presented.

One notable difference from formal thought disorder research is that disorganization is typically only exacerbated in unpleasant valence conditions; in this study, however, fluency also decreased in psychometric schizotypy in the pleasant valence condition. One explanation could be that those with psychometric schizotypy—similar to people with schizophrenia (Cohen \& Minor, 2010)_experience greater negative affect than non-schizotypy groups when pleasant stimuli are introduced. Negative affect constricts attention, which could have a deleterious effect on fluency performance in the pleasant valence condition (Lazar, Kaplan, Sternberg, \& Lubow, 2012). Although we did not conduct pre- or post-test assessments, examining the role of positive and negative affect following the administration of affective valence fluency tests is an important avenue for future research.

Minor support was observed for hypotheses concerning cognitive load and schizotypy traits. Regarding cognitive load, both schizotypy and non-schizotypy groups performed significantly worse in this condition compared to baseline. Counter to expectations, however, no group differences were found. This may be due to the 1-back task not providing enough of a distractor for subjects. Future studies should increase task difficulty when examining the cognitive load effect. Findings regarding schizotypy traits partially supported previous research in schizophrenia and schizotypy suggesting that those with predominantly positive symptoms/ traits tend to be more reactive when load is introduced compared to individuals with predominantly negative symptoms/ traits (Docherty \& Hebert, 1997; Minor \& Cohen, 2010; 
Myin-Germeys et al., 2002; Schwartz \& Myers, 1977). Findings must be replicated, however, as positive traits were only associated with category fluency performance in one of four conditions. The undergraduate sample serves as both a strength and potential limitation. Undergraduate samples are commonly recruited in psychometric schizotypy studies (Chapman, Chapman, Kwapil, Eckblad, \& Zinser, 1994; Gooding et al., 2005; Kerns, 2007; Kwapil et al., 1997; Minor \& Cohen, 2012; Minor et al., 2014), and include people in the peak age of onset while allowing researchers to examine constructs without confounding factors typically found in patient populations (e.g., antipsychotic medications; Chapman et al., 1994). However, participation in college courses suggests a higher level of functioning compared to other individuals on the schizophrenia-spectrum. Future research should examine category fluency performance in people recruited from community samples and results should be compared to those in undergraduate cohorts. A second limitation of this study is that positive and negative affect was not assessed before or after pleasant and unpleasant category fluency tests. Pre- and post-test assessments would aid in demonstrating the role of affective load on performance across each test and should be administered in future studies.

In sum, category fluency performance in psychometric schizotypy appears to be influenced by affective load. Whereas people with schizophrenia exhibit fluency deficits in baseline and affective conditions (Rossell, 2006), significant differences in psychometric schizotypy only emerged once pleasant and unpleasant stimuli were introduced. In contrast to affective load, cognitive load had little impact on fluency when schizotypy and non-schizotypy performance was compared. Within schizotypy, positive and disorganized traits were associated with decreased fluency; however, findings were inconsistent across conditions. Based on our findings, future studies should examine whether category fluency deficits predict future 
conversion to psychosis in psychometric schizotypy - particularly in those who are more reactive to affective stimuli. If so, these deficits may have utility as risk markers in both clinical high-risk (see Becker et al., 2010) and psychometric schizotypy populations. 


\section{References}

Becker, H. E., Nieman, D. H., Dingemans, P. M., van de Fliert, J. R., De Haan, L., \& Linszen, D. H. (2010). Verbal fluency as a possible predictor for psychosis. European Psychiatry, 25, 105-110. doi: 10.1016/j.eurpsy.2009.08.003

Bokat, C. E., \& Goldberg, T. E. (2003). Letter and category fluency in schizophrenic patients: a meta-analysis. Schizophrenia Research, 64, 73-78. Retrieved from http://www.web.b.ebscohost/ehost

Chapman, L. J., Chapman, J. P., Kwapil, T. R., Eckblad, M., \& Zinser, M. C. (1994). Putatively psychosis-prone subjects 10 years later. Journal of Abnormal Psychology, 111, 186-191. Retrieved from http://www.web.b.ebscohost/ehost

Chun, C. A., Minor, K. S., \& Cohen, A. S. (2013). Neurocognition in psychometrically defined college Schizotypy samples: we are not measuring the "right stuff". Journal of the International Neuropsychological Society, 19, 324-337. doi: $10.1017 / \mathrm{S} 135561771200152 \mathrm{X}$

Cohen, A. S., Matthews, R. A., Najolia, G. M., \& Brown, L. A. (2010). Toward a more psychometrically sound brief measure of schizotypal traits: introducing the SPQ-Brief Revised. Journal of Personality Disorders, 24, 516-537. doi: 10.1521/pedi.2010.24.4.516

Cohen, A. S., \& Minor, K. S. (2010). Emotional experience in patients with schizophrenia revisited: meta-analysis of laboratory studies. Schizophrenia Bulletin, 36, 143-150. doi: $10.1093 / \mathrm{schbul} / \mathrm{sbn} 061$

Cohen, A. S., \& Najolia, G. M. (2011). Birth characteristics and schizotypy: Evidence of a potential “second hit." Journal of Psychiatric Research, 45, 955-961. doi: 10.1016/j.jpsychires.2010.12.006. 
Docherty, N. M., \& Hebert, A. S. (1997). Comparative affective reactivity of different types of communication disturbances in schizophrenia. Journal of Abnormal Psychology, 106, 325-330. Retrieved from http://www.web.b.ebscohost/ehost

Eastvold, A. D., Heaton, R. K., \& Cadenhead, K. S. (2007). Neurocognitive deficits in the (putative) prodrome and first episode of psychosis. Schizophrenia Research, 93, 266-277. doi: 10.1016/j.schres.2007.03.013

Gooding, D. C., Tallent, K. A., \& Matts, C. W. (2005). Clinical status of at-risk individuals 5 years later: further validation of the psychometric high-risk strategy. Journal of Abnormal Psychology, 114, 170-175. doi: 10.1037/0021-843X.114.1.170

Harvey, P. D., Reichenberg, A., Romero, M., Granholm, E., \& Siever, L. J. (2006). Dual-task information processing in schizotypal personality disorder: evidence of impaired processing capacity. Neuropsychology, 20, 453-460. doi: 10.1037/0894-4105.20.4.453

Hawkins, K. A., Addington, J., Keefe, R. S., Christensen, B., Perkins, D. O., Zipurksy, R., . . . McGlashan, T. H. (2004). Neuropsychological status of subjects at high risk for a first episode of psychosis. Schizophrenia Research, 67, 115-122. doi:

10.1016/j.schres.2003.08.007

Kerns, J. G. (2007). Experimental manipulation of cognitive control processes causes an increase in communication disturbances in healthy volunteers. Psychological Medicine, 37, 9951004. doi: $10.1017 / \mathrm{S} 0033291706009718$

Kerns, J. G., \& Becker, T. M. (2008). Communication disturbances, working memory, and emotion in people with elevated disorganized schizotypy. Schizophrenia Research, 100, 172-180. doi: 10.1016/j.schres.2007.11.005 
Kerns, J. G., \& Berenbaum, H. (2002). Cognitive impairments associated with formal thought disorder in people with schizophrenia. Journal of Abnormal Psychology, 111, 211-224. Retrieved from http://www.web.b.ebscohost/ehost

Kwapil, T. R., Miller, M. B., Zinser, M. C., Chapman, J., \& Chapman, L. J. (1997). Magical ideation and social anhedonia as predictors of psychosis proneness: a partial replication. Journal of Abnormal Psychology, 106, 491-495. Retrieved from http://www.web.b.ebscohost/ehost

Lazar, J., Kaplan, O., Sternberg, T., \& Lubow, R. E. (2012). Positive and negative affect produce opposing task-irrelevant stimulus preexposure effects. Emotion, 12, 591-604. doi: $10.1037 / \mathrm{a} 0024867$

Lenzenweger, M. F. (2006). Schizotaxia, schizotypy, and schizophrenia: Paul E. Meehl's blueprint for the experimental psychopathology and genetics of schizophrenia. Journal of Abnormal Psychology, 115, 195-200. doi: 10.1037/0021-843X.115.2.195

Meehl, P.E. (1990). Toward an integrated theory of schizotaxia, schizotypy, and schizophrenia. Journal of Personality Disorders, 4, 1-99. Retrieved from http://www.web.b.ebscohost/ehost

Minor, K. S., \& Cohen, A. S. (2010). Affective reactivity of speech disturbances in schizotypy. Journal of Psychiatric Research, 44, 99-105. doi: 10.1016/j.jpsychires.2009.06.005

Minor, K. S., \& Cohen, A. S. (2012). The role of atypical semantic activation and stress in odd speech: implications for individuals with psychometrically defined schizotypy. Journal of Psychiatric Research, 46, 1231-1236. doi: 10.1016/j.jpsychires.2012.06.001 
Minor, K. S., Cohen, A. S., Weber, C. R., \& Brown, L. A. (2011). The relationship between atypical semantic activation and odd speech in schizotypy across emotionally evocative conditions. Schizophrenia Research, 126, 144-149. doi: 10.1016/j.schres.2010.06.016

Minor, K. S., Firmin, R. L., Bonfils, K. A., Chun, C. A., Buckner, J. D., \& Cohen, A. S. (2014). Predicting creativity: The role of psychometric schizotypy and cannabis use in divergent thinking. Psychiatry Research, 220, 205-210. doi: 10.1016/j.psychres.2014.08.044

Morrens, M., Krabbendam, L., Bak, M., Delespaul, P., Mengelers, R., Sabbe, B., ... MyinGermeys, I. (2007). The relationship between cognitive dysfunction and stress sensitivity in schizophrenia: A replication study. Social Psychiatry and Psychiatric Epidemiology, 42, 284-287. doi: 10.1007/s00127-007-0170-7

Myin-Germeys, I., Krabbendam, L., Jolles, J., Delespaul, P. A., \& van Os, J. (2002). Are cognitive impairments associated with sensitivity to stress in schizophrenia? An experience sampling study. American Journal of Psychiatry, 159, 443-449. Retrieved from http://www.web.b.ebscohost/ehost

Myin-Germeys, I., \& van Os, J. (2007). Stress-reactivity in psychosis: evidence for an affective pathway to psychosis. Clinical Psychology Review, 27, 409-424. doi:

10.1016/j.cpr.2006.09.005

Neill, E., Gurvich, C., \& Rossell, S. L. (2014). Category fluency in schizophrenia research: is it an executive or semantic measure? Cognitive Neuropsychiatry, 19, 81-95. doi: $10.1080 / 13546805.2013 .807233$

Nuechterlein, K. H., Pashler, H. E., \& Subotnik, K. L. (2006). Translating basic attentional paradigms to schizophrenia research: reconsidering the nature of the deficits. 
Developmental Psychopathology, 18, 831-851. Retrieved from

http://www.web.b.ebscohost/ehost

Raine, A. (1991). The SPQ: a scale for the assessment of schizotypal personality based on DSMIII-R criteria. Schizophrenia Bulletin, 17, 555-564. Retrieved from http://www.web.b.ebscohost/ehost

Rossell, S. L. (2006). Category fluency performance in patients with schizophrenia and bipolar disorder: The influence of affective categories. Schizophrenia Research, 82, 135-138. doi: 10.1016/j.schres.2005.10.013

Ruthruff, E., Pashler, H. E., \& Klaassen, A. (2001). Processing bottlenecks in dual-task performance: structural limitation or strategic postponement? Psychonomic Bulletin \& Review, 8, 73-80. Retrieved from http://www.web.b.ebscohost/ehost

Schwartz, C. C., \& Myers, J. K. (1977). Life events and schizophrenia. II. Impact of life events and symptom configuration. Archives of General Psychiatry, 34, 1240-1245. Retrieved from http://www.web.b.ebscohost/ehost

Sweller, J. (1983). Control mechanisms in problem solving. Memory and Cognition, 11, 32-40. Retrieved from http://www.web.b.ebscohost/ehost

Szoke, A., Trandafir, A., Dupont, M. E., Meary, A., Schurhoff, F., \& Leboyer, M. (2008). Longitudinal studies of cognition in schizophrenia: meta-analysis. British Journal of Psychiatry, 192, 248-257. doi: 10.1192/bjp.bp.106.029009

van Merrienboer, J. J., \& Sweller, J. (2010). Cognitive load theory in health professional education: design principles and strategies. Medical Education, 44, 85-93. doi: 10.1111/j.1365-2923.2009.03498.x 
Table 1. Demographics and verbal intelligence of schizotypy and non-schizotypy groups.

\begin{tabular}{|l|c|c|c|c|c|}
\hline & \multicolumn{2}{|c|}{ Schizotypy $(n=42)$} & \multicolumn{2}{c|}{ Non-schizotypy $(n=38)$} & \\
\hline \multicolumn{1}{|c|}{ Variable } & Mean & SD & Mean & SD & $p$ \\
\hline Age & 19.48 & 1.61 & 18.94 & 1.09 & 0.09 \\
\hline Gender (\% female) & \multicolumn{2}{|c|}{$79 \%$} & \multicolumn{2}{c|}{$71 \%$} & 0.44 \\
\hline Ethnicity (\% caucasian) & \multicolumn{2}{|c|}{$89 \%$} & $83 \%$ & 0.60 \\
\hline Verbal intelligence & 105.15 & 10.66 & 104.19 & 11.75 & 0.71 \\
\hline
\end{tabular}


Table 2. Comparison of schizotypy and non-schizotypy groups on category fluency tests.

\begin{tabular}{|c|c|c|c|c|}
\hline Category Fluency Test & \multicolumn{2}{|c|}{ Schizotypy $(n=42)$} & \multicolumn{2}{|c|}{ Non-schizotypy $(n=38)$} \\
\hline Baseline & Mean & SD & Mean & SD \\
\hline Uncorrected $^{\mathrm{a}}$ & 13.05 & 3.88 & 13.05 & 3.85 \\
\hline Corrected & 13.76 & 3.86 & 13.92 & 3.66 \\
\hline \multicolumn{5}{|l|}{ Cognitive Load } \\
\hline Uncorrected $^{b}$ & 11.26 & 4.05 & 11.24 & 3.67 \\
\hline Corrected & 12.31 & 3.76 & 12.08 & 3.72 \\
\hline \multicolumn{5}{|l|}{ Affective } \\
\hline Pleasant & $13.33^{*}$ & 3.84 & 15.00 & 4.65 \\
\hline Unpleasant & $9.10 *$ & 4.26 & 10.92 & 4.13 \\
\hline
\end{tabular}

Note: ${ }^{*} p<.05$ (Between-group comparison); SD: standard deviation; ${ }^{\mathrm{a} F r u i t}$ Mean (SD): Schizotypy $(n=22)=13.50$ (3.57), Non-schizotypy $(n=16)=14.56$ (3.12); Vegetable Mean (SD): Schizotypy $(n=20)=12.55$ (4.24), Nonschizotypy $(n=22)=11.95(4.02){ }^{b}$ Fruit Mean (SD): Schizotypy $(n=20)=12.95(3.75)$, Non-schizotypy $(n=22)=$ 11.50 (3.32); Vegetable Mean (SD): Schizotypy $(n=22)=9.73(3.76)$, Non-schizotypy $(n=16)=10.88(4.19)$.

URL: http://mc.manuscriptcentral.com/pcnp Email: reviews@psypress.co.uk 
Figure 1. Category fluency performance on experimental compared to baseline category fluency tests across schizotypy and non-schizotypy groups.

1

2

3

4

5

6

7

8

9

10

11

12

13

14

15

16

17

18

19

20

21

22

23

24

25

26

27

28

29

30

31

32

33

34

35

36

37

38

39

40

41

42

43

44

45

46

47

48

49

50

51

52

53

54

55

56

57

58

59

60

URL: http://mc.manuscriptcentral.com/pcnp Email: reviews@psypress.co.uk 


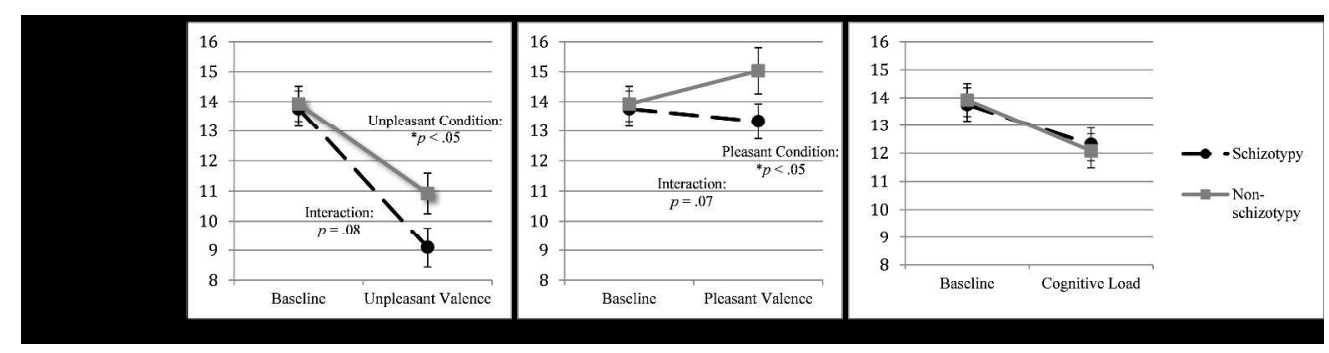

$1632 \times 413 \mathrm{~mm}(96 \times 96 \mathrm{DPI})$ 